\title{
Increase of Sodium Channels (Nav 1.8 and Nav 1.9) in Rat Dorsal Root Ganglion Neurons Exposed to Autologous Nucleus Pulposus
}

\author{
Kazuyuki Watanabe ${ }^{1,2}$, Karin Larsson ${ }^{1}$, Björn Rydevik ${ }^{1}$, Shin-ichi Konno ${ }^{2}$, Claes Nordborg ${ }^{3}$ and \\ Kjell Olmarker ${ }^{*}, 4$
}

\author{
${ }^{I}$ Department of Orthopaedics, Institute of Clinical Sciences, Sahlgrenska Academy, University of Gothenburg, \\ Gothenburg, Sweden \\ ${ }^{2}$ Department of Orthopaedic Surgery, Fukushima Medical University, School of Medicine, Fukushima, Japan \\ ${ }^{3}$ Department of Pathology, Sahlgrenska Academy, University of Gothenburg, Gothenburg, Sweden \\ ${ }^{4}$ Musculoskeletal Research, Department of Medical Chemistry and Cell Biology, Institute of Biomedicine, Sahlgrenska \\ Academy, University of Gothenburg, Gothenburg, Sweden
}

\begin{abstract}
Purpose: It has been assumed that nucleus pulposus-induced activation of the dorsal root ganglion (DRG) may be related to an activation of sodium channels in the DRG neurons. In this study we assessed the expression of Nav 1.8 and Nav 1.9 following disc puncture.

Method: Thirty female Sprague-Dawley rats were used. The L4/L5 disc was punctured by a needle $(\mathrm{n}=12)$ and compared to a sham group without disc puncture $(n=12)$ and a naive group $(n=6)$. At day 1 and 7 , sections of the left L4 DRG were immunostained with anti-Nav 1.8 and Nav 1.9 antibodies.

Result: At day 1 after surgery, both Nav 1.8-IR neurons and Nav 1.9-IR neurons were significantly increased in the disc puncture group compared to the sham and naive groups $(\mathrm{p}<0.05)$, but not at day 7 .

Conclusion: The findings in the present study demonstrate a neuronal mechanism that may be of importance in the pathophysiology of sciatic pain in disc herniation.
\end{abstract}

Keywords: DISC herniation, dorsal root ganglion, rat, sciatica, spine, voltage-gated sodium channel.

\section{INTRODUCTION}

Application of nucleus pulposus on nerve tissue has recently been linked to the occurrence of pain-related behavior in various experimental models $[1,2]$. However, it seems likely that pain only appears, or is at least more pronounced, if exposure of the nerve tissue to nucleus pulposus is combined with mechanical deformation of the nerve tissue $[1,2]$. This may suggest that nucleus pulposus sensitizes the nerve tissue to produce nerve root pain when the nerve tissue is deformed mechanically. There is also clinical evidence indicating that mechanical stimulation of a nerve root not previously exposed to nucleus pulposus results in only slight discomfort, whereas mechanical stimulation of a nerve root exposed to nucleus pulposus instead reproduces the sciatic pain [3]. Nucleus pulposus application on nerve tissue has also experimentally been found to induce electrophysiological changes such as activation of dorsal root ganglion (DRG) neurons [4] and ectopic discharge of the spinal dorsal horn neurons [5] as well as increased expression of sodium channels in neurons

*Address correspondence to this author at the Musculoskeletal Research, Department of Medical Chemistry and Cell Biology, Institute of Biomedicine, Sahlgrenska Academy, University of Gothenburg, Gothenburg, Sweden; Tel: +46-31-7866660; E-mail. kjell.olmarker@gu.se in the DRG [6]. These data together suggest that nucleus pulposus induces hyperexcitability in DRG neurons.

Ion channels are very important in neuronal activity in generating and conducting action potential. Especially, sodium channels have been in focus in pain research, since the sodium channel blocker lidocaine is very useful clinically to block pain [7]. There are nine distinct voltage-gated sodium channel $\alpha$ subunits; Nav 1.1-1.9 [8]. Adult DRG sensory neurons may express a combination of Nav 1.1, Nav 1.6, Nav 1.7, Nav 1.8 and Nav 1.9 sodium channels $[9,10]$. Nav 1.9 is mostly expressed in neurons with unmyelinated axons (C-fibers) [11, 12] and Nav 1.8 in neurons with unmyelinated and myelinated axons (A-fibers) [11, 13], and all these types of neurons are associated with transmission of nociceptive impulse. There are many reports about changes of these sodium channels after nerve injury and inflammation. However, it is unclear whether these sodium channels in the DRG neurons may be upregulated after lumbar disc herniation. The purpose of this study was to investigate the expression of Nav 1.8 and Nav 1.9 in DRG neurons exposed to nucleus pulposus using immunohistochemical methods.

\section{MATERIALS AND METHODOLOGY}

Thirty female Sprague-Dawley rats with an average body weight of 200-250g were housed in groups with free access 
to food (B\&K Rat/mouse standard, BeeKay feeds \& beddings, Sollentuna, Stockholm) and tap water. Temperature was kept at $21^{\circ} \mathrm{C}$, light schedule was 12 hours daylight starting at 6:00 a.m. and 12 hours darkness starting at 6:00 p.m., and the humidity was kept at 50\%. The experimental protocol was approved by the local animal research ethics committee.

Surgical protocol [1]: The rats were anesthetized by inhalation of isoflurane. Through a midline incision, the thoracolumbar fascia was incised just left to the spinous processes of the $4^{\text {th }}$ and the $5^{\text {th }}$ lumbar vertebrae. The erector spinae muscle was gently moved laterally to expose the left facet joint between the $4^{\text {th }}$ and the $5^{\text {th }}$ lumbar vertebrae. The joint, including articular processes, was carefully removed. By this procedure, the ligamentum flavum, the left L4 DRG and the intervertebral disc could easily be identified. To form a control group for the surgical exposure of the spinal canal, no additional procedures were undertaken in twelve rats (sham group: $n=12$ ). To perform a disc herniation, a 25 Gauge needle was used to puncture the exposed disc (NP group: $n=12$ ). By gently injecting some air through the needle there was a leakage of nucleus pulposus out to the DRG. To ensure contact between the nucleus pulposus and the nervous tissue the obtained nucleus pulposus was transferred with the tip of the needle to the $4^{\text {th }}$ lumbar nerve root and DRG. No surgical intervention was performed in the Naïve group $(n=6)$.

\section{Immunohistochemistry}

At day 1 and 7 after surgery, rats were deeply anaesthetized with an intraperitoneal injection of $0.2 \mathrm{ml}$ pentobarbital $(50 \mathrm{mg} / \mathrm{ml})$ and underwent intracardiac perfusion with $200 \mathrm{ml}$ saline followed by $200 \mathrm{ml}$ of Histofix (4\% formalin)(Histolab Products AB, Sweden). The left L4 DRGs were removed and postfixed for 24 hours. The DRGs were dehydrated, embedded in paraffin, and cut into transverse $4.5 \mu \mathrm{m}$ sections. The sections were mounted on slides, deparaffinized with xylene, rehydrated, and processed immunohistochemically. The endogenous peroxidase in the sections was suppressed with hydrogen peroxidase (PeroxiDazed1) (Biocare Medical, Concord, CA, USA) for $5 \mathrm{~min}$ and then washed in Tris Buffered Saline (TBS) (Biocare Medical, Concord, CA, USA). For reducing non-specific binding, the sections were incubated with goat serum for $30 \mathrm{~min}$ at room temperature. The sections were then incubated with the primary antibodies, rabbit antiNav 1.8(Chemicon, Temecula, CA, USA), in a dilution of 1:100 or rabbit anti-Nav 1.9(Chemicon, Temecula, CA, USA), in a dilution of 1:200, for $30 \mathrm{~min}$ at room temperature. The incubated sections were washed in TBS and incubated with secondary antibody MACH2 $2^{\mathrm{TM}}$ Polymer-HRP Conjugate (Goat Anti-Rabbit) (Biocare Medical, USA) for $30 \mathrm{~min}$ at room temperature. For visualizing the immunohistochemical staining, Betazoid DABChromagen Kit (Biocare Medical, USA) was used. Examination of the sections was performed with light microscopy. All analysis were performed in a blinded fashion.

\section{Data Analysis}

The total number of neurons and Nav -like immunoreactive (IR) neurons with visible nucleus in one complete transverse section was counted. Neurons showing higher intensity than background were considered positive using imaging analysis software (NIH image $\mathrm{J}$ ). The cross sectional area of the neurons was also examined to categorize to small sized neurons $\left(<600 \mu \mathrm{m}^{2}\right)$, medium sized $\left(600-1200 \mu \mathrm{m}^{2}\right)$, and large sized neurons $\left(>1200 \mu \mathrm{m}^{2}\right)$. To be able to observe as many neurons as possible, we picked sections from the central one third of DRG as defined by the shape of the DRG. Six randomly selected sections were examined per rat. The percentage of Nav -IR neurons of the total number of neurons was calculated. The results are expressed as the percentage of Nav -IR neurons in the DRGs (mean \pm standard error of the mean).

Statistical analysis was performed using ANOVA and Fisher's PLSD. P-values less than 0.05 were considered significant.

\section{RESULTS}

Both Nav 1.8 and Nav 1.9 were stained positively in the somata of the DRG neurons in all experimental groups, especially in the small-sized neurons $\left(<600 \mu \mathrm{m}^{2}\right)($ Figs. 1, 2).

At $1^{\text {st }}$ day after surgery, the percentages of Nav 1.8-IR neurons significantly increased in the disc puncture group compared with the Sham and Naïve groups in small sized neurons $(\mathrm{p}<0.05)$ (Fig. 3). There were no significant differences in medium and large sized neurons regarding Nav 1.8 expression. Nav 1.9-IR neurons in small sized neurons significantly increased in the disc puncture group compared with the naive groups $(\mathrm{p}<0.05)$ and there were also significant differences between sham and naïve group $(\mathrm{p}<0.05)$. There were no significant differences regarding Nav 1.9-IR neurons in the disc puncture group compared with sham group $(\mathrm{p}<0.1)$, (Fig. 4). There were also no significant differences in medium and large sized neurons. No significant differences were found among the three groups at 7 days after surgery in both Nav 1.8 and Nav 1.9IR neurons (Figs. 3, 4).

\section{DISCUSSION}

Leakage of nucleus pulposus from a lumbar disc to the dorsal root ganglion (DRG) induced transient increases of Nav 1.8 and Nav 1.9-IR neurons in the affected DRGs. In a corresponding model, pain-related behavior was only seen at one day postoperatively, but not 7 days after surgery [1]. It is known that nucleus pulposus can induce injury of axons [14] and inflammatory reactions around the nerve tissue [15]. Therefore, the transient increase in expression of Nav 1.8 and Nav 1.9 in the present study might theoretically have been influenced by neuronal damage as well as inflammatory responses, as induced by nucleus pulposus. Such changes of sodium channels in DRG neurons can be expected to be related to the electrophysiological properties of the neurons, including sensitization of DRG neurons. This could explain the pain mechanisms induced by nucleus pulposus. Nav 1.8 has been reported to be very important in pain sensation since a knock out of this channel [16] or blockage of this channel by specific blockers [17] showed a dramatic improvement of inflammatory pain in animals. A knock out of Nav 1.8 [18] and blockage of Nav $1.8[17,19,20]$ has been 

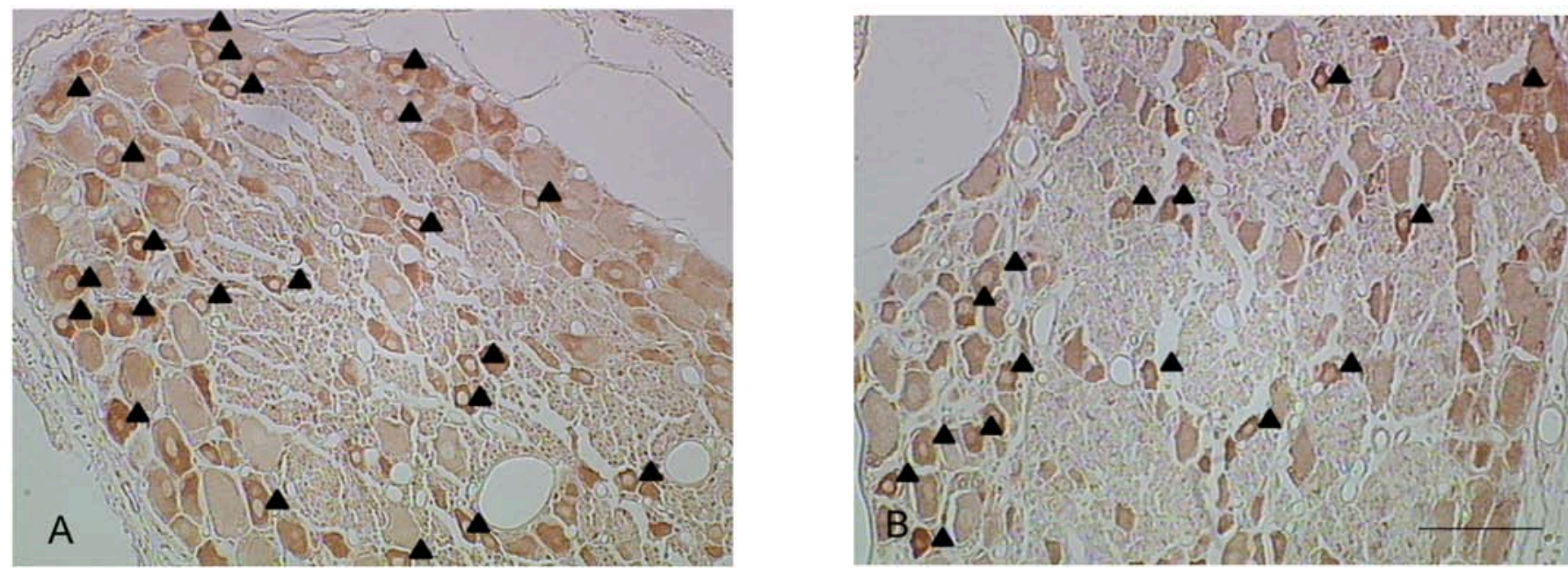

Fig. (1). Representative pictures of Nav 1.8-IR neurons in the disc puncture group (A) and the sham group (B). Small and medium but not large sized neurons showed immune reactivity for Nav 1.8 ( $\boldsymbol{\Lambda}$ : Nav 1.8-IR neurons, Bar: $100 \mu \mathrm{m})$.
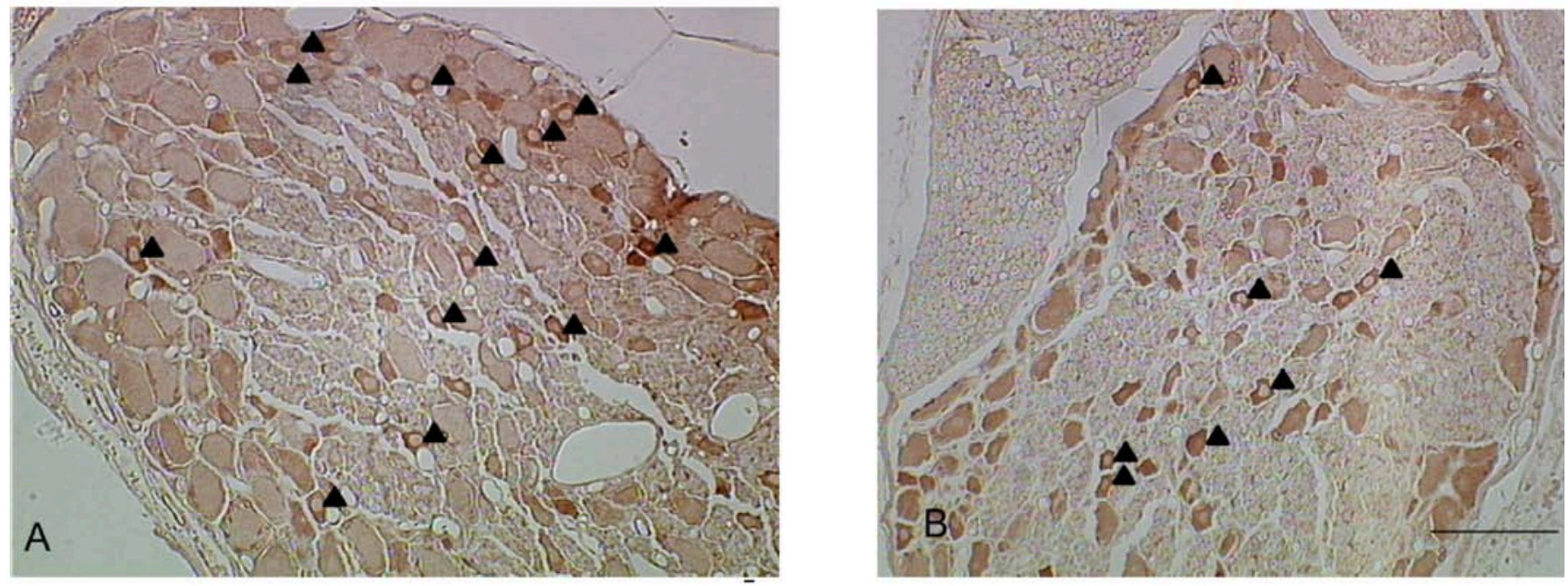

Fig. (2). Representative pictures of Nav 1.9-IR neurons in the disc puncture model (A) and the sham group (B). Most of the Nav 1.9-IR neurons were small size neurons ( $\boldsymbol{\Lambda}$ : Nav 1.9-IR neurons, Bar: $100 \mu \mathrm{m}$ ).

found to also attenuate neuropathic pain. Neuropathic pain induced by partial axotomy induced a decrease of Nav 1.8 RNA and protein [21]. On the other hand, inflammatory pain induced by carrageenan caused an increase of Nav 1.8 [22]. Therefore, increase of Nav 1.8 induced by nucleus pulposus might follow the inflammatory effects of nucleus pulposus.

Nav 1.9 mRNA is likewise upregulated by inflammation and downregulated by axotomy [10]. Nav 1.9 null mice showed a decrease in inflammatory pain behavior but not in neuropathic pain [23]. Therefore, Nav 1.9 may be more closely related to inflammatory pain transmission than to neuropathic pain. Nav 1.9 current was increased by an inflammatory agent such as PGE2 [24]. Disc tissue harvested from the patients with sciatica contained many pro inflammatory mediators including PGE2 [25]. Therefore, in the model in the present study, there is a possibility that Nav 1.9 was increased by inflammatory reactions induced by nucleus pulposus. This, however, remains to be clarified in future studies.

One discrepancy between the present and previous studies was that upregulation was not found seven days after surgery in our study, whereas pain behavior changes, although less pronounced than at day 1 or 3 , may still be seen at day seven $[1,2]$. In the present study ion-channels were only studied in the dorsal root ganglion neurons and it is suspected that there may be a similar upregulation in the axons of the nerve roots that may sustain more than seven days. Also other modes of injury, such as simultaneous nucleus pulposus-exposure and mechanical deformation would be of certain interest, as well as the treatment with sodium channel-inhibitors. This will be assessed in future studies.

\section{CONCLUSION}

Clinically, sciatic pain induced by lumbar disc herniation is sometimes so pronounced that it is difficult to control the pain by presently available conservative treatment modalities such as NSAIDs, or nerve root block with local anesthetics and steroids. New strategies are needed to provide an alternative to surgery in such cases. Since the present immunohistochemical study indicates that sodium channels in DRG neurons may be increased by nucleus pulposus- 


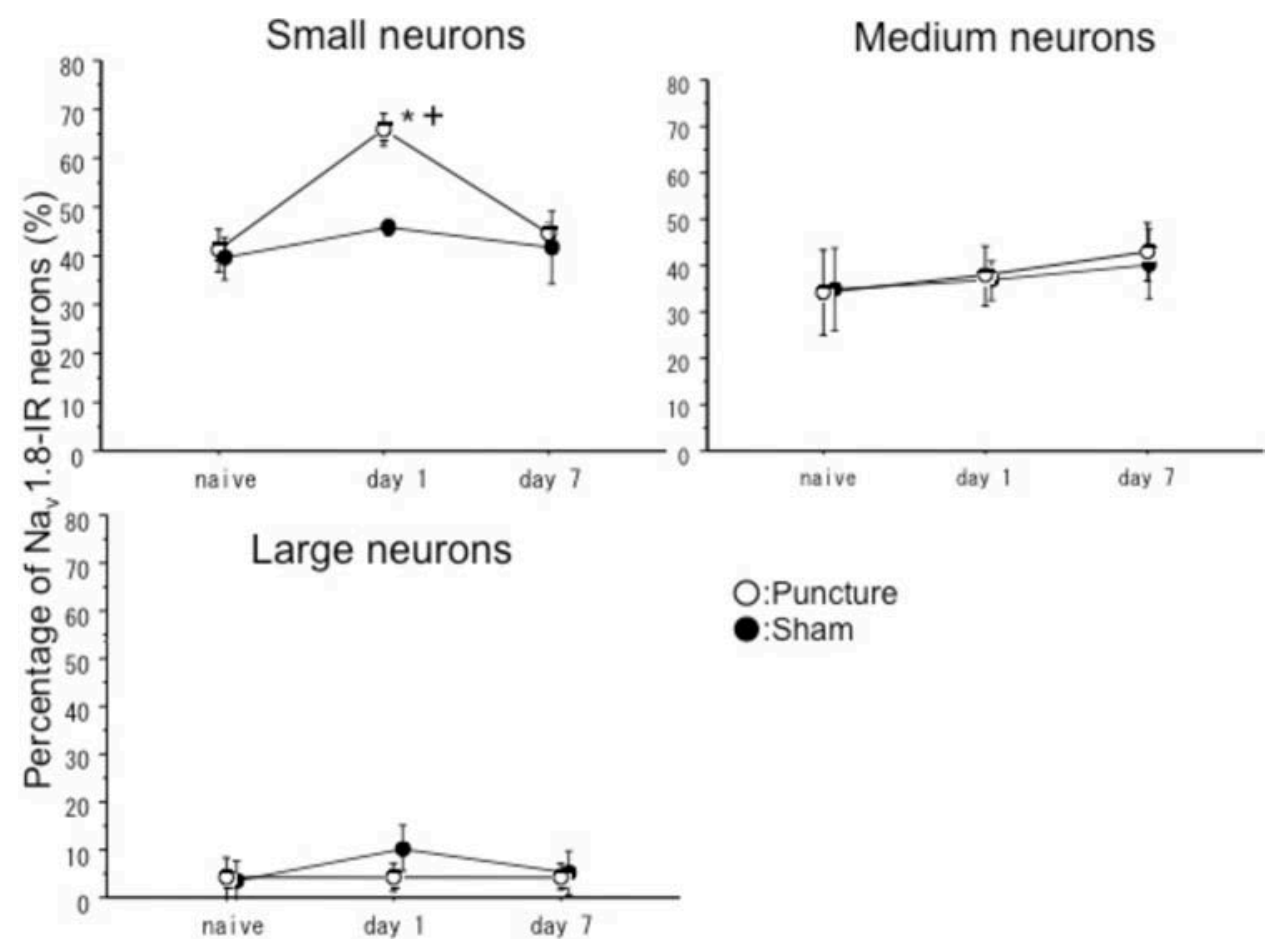

Fig. (3). These graphs show percentages of Nav 1.8-IR neurons in small sized $\left(<600 \mu \mathrm{m}^{2}\right)$, medium sized $\left(600-1200 \mu \mathrm{m}^{2}\right)$ and large sized neurons $\left(>1200 \mu \mathrm{m}^{2}\right)$. There were significant differences between puncture and sham groups, and puncture and naïve groups in small neurons at Day 1. There were no significant differences in medium and large sized neurons. There were no significant differences among three groups at Day 7. * $\mathrm{p}<0.05$ compared with naïve group, $+\mathrm{p}<0.05$ compared sham group.

Small neurons

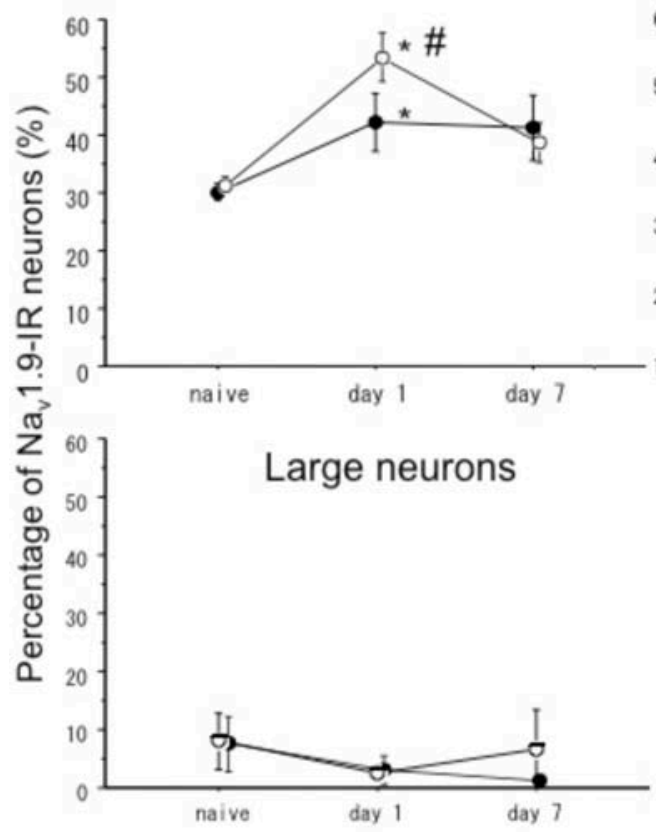

Medium neurons

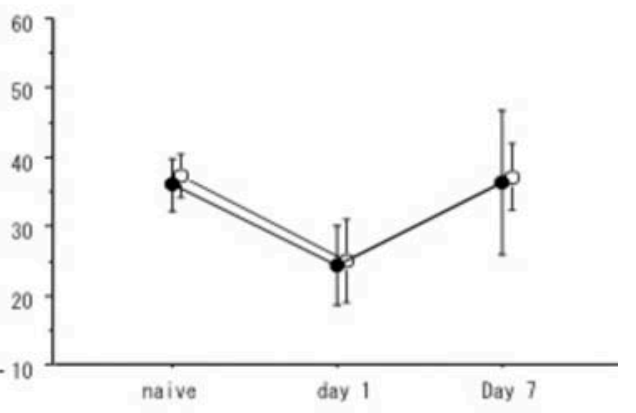

O:Puncture

:Sham

Fig. (4). These graphs show percentages of Nav 1.9-IR neurons in small sized neurons $\left(<600 \mu \mathrm{m}^{2}\right)$, medium sized $\left(600-1200 \mu \mathrm{m}^{2}\right)$ and large sized neurons $\left(>1200 \mu \mathrm{m}^{2}\right)$. There were significant differences between puncture and naïve group, and sham and naïve group in small neurons at Day 1. The percentage of Nav 1.8-IR neurons tend to be higher in small sized neurons in puncture group compared with sham group at Day 1. There were no significant differences in medium and large sized neurons. There were no significant differences among three groups at Day 7. ${ }^{*} \mathrm{p}<0.05$ compared with naïve group, \# $\mathrm{p}<0.1$ compared with sham group.

exposure, specific blockages of neuronal sodium channels might offer such an alternative.

\section{CONFLICT OF INTEREST}

The author confirms that this article content has no conflict of interest. 


\section{ACKNOWLEDGEMENTS}

This work was supported by grants from the Swedish Research Council (521-2007-2956), the Gothenburg Medical Society, AFA Insurance, the Ollie and Elof Ericsson Foundation for Scientific Research, Stiftelsen Olle Engkvist byggmästare, the IngaBritt and Arne Lundberg Research Foundation, the Spine Society of Europe Task force on research and the Felix Neubergh Foundation.

\section{REFERENCES}

[1] Olmarker K, Myers RR. Pathogenesis of sciatic pain: role of herniated nucleus pulposus and deformation of spinal nerve root and dorsal root ganglion. Pain 1998; 78(2): 99-105.

[2] Kawakami M, Tamaki $\mathrm{T}$, Hayashi $\mathrm{N}$, et al. Mechanical compression of the lumbar nerve root alters pain-related behaviors induced by the nucleus pulposus in the rat. J Orthop Res 2000; 18(2): 257-64.

[3] Kuslich SD, Ulstrom CL, Michael CJ. The tissue origin of low back pain and sciatica: a report of pain response to tissue stimulation during operations on the lumbar spine using local anesthesia. Orthop Clin North Am 1991; 22(2): 181-7.

[4] Takebayashi T, Cavanaugh JM, Cuneyt Ozaktay A, Kallakuri S, Chen C. Effect of nucleus pulposus on the neural activity of dorsal root ganglion. Spine 2001; 26(8): 940-5.

[5] Anzai H, Hamba M, Onda A, Konno S, Kikuchi S. Epidural application of nucleus pulposus enhances nociresponses of rat dorsal horn neurons. Spine 2002; 27(3): E50-5.

[6] Chen C, Cavanaugh JM, Song Z, Takebayashi T, Kallakuri S, Wooley PH. Effects of nucleus pulposus on nerve root neural activity, mechanosensitivity, axonal morphology, and sodium channel expression. Spine (Phila Pa 1976) 2004; 29(1): 17-25.

[7] Gold MS. Tetrodotoxin-resistant Na+ currents and inflammatory hyperalgesia. Proc Natl Acad Sci USA 1999; 96(14): 7645-9.

[8] Goldin AL, Barchi RL, Caldwell JH, et al. Nomenclature of voltage-gated sodium channels. Neuron 2000; 28(2): 365-8.

[9] Black JA, Dib-Hajj S, McNabola K, et al. Spinal sensory neurons express multiple sodium channel alpha-subunit mRNAs. Brain Res Mol Brain Res 1996; 43(1-2): 117-31.

[10] Dib-Hajj SD, Tyrrell L, Black JA, Waxman SG. NaN, a novel voltage-gated $\mathrm{Na}$ channel, is expressed preferentially in peripheral sensory neurons and downregulated after axotomy. Proc Natl Acad Sci USA 1998; 95(15): 8963-8.

[11] Amaya F, Decosterd I, Samad TA, et al. Diversity of expression of the sensory neuron-specific TTX-resistant voltage-gated sodium ion channels SNS and SNS2. Mol Cell Neurosci 2000; 15(4): 33142 .

[12] Fang X, Djouhri L, Black JA, Dib-Hajj SD, Waxman SG, Lawson $\mathrm{SN}$. The presence and role of the tetrodotoxin-resistant sodium channel $\mathrm{Na}(\mathrm{v}) 1.9(\mathrm{NaN})$ in nociceptive primary afferent neurons. $\mathbf{J}$ Neurosci 2002; 22(17): 7425-33.

[13] Djouhri L, Fang X, Okuse K, Wood JN, Berry CM, Lawson SN. The TTX-resistant sodium channel Nav 1.8 (SNS/PN3): expression and correlation with membrane properties in rat nociceptive primary afferent neurons. J Physiol 2003; 550(Pt 3): 739-52.

[14] Olmarker K, Nordborg C, Larsson K, Rydevik B. Ultrastructural changes in spinal nerve roots induced by autologous nucleus pulposus. Spine 1996; 21(4): 411-4.

[15] Olmarker K, Blomquist J, Stromberg J, Nannmark U, Thomsen P, Rydevik B. Inflammatogenic properties of nucleus pulposus. Spine 1995; 20(6): 665-9.

[16] Joshi SK, Mikusa JP, Hernandez G, et al. Involvement of the TTXresistant sodium channel Nav 1.8 in inflammatory and neuropathic, but not post-operative, pain states. Pain 2006; 123(1-2): 75-82.

[17] Jarvis MF, Honore P, Shieh CC, et al. A-803467, a potent and selective Nav 1.8 sodium channel blocker, attenuates neuropathic and inflammatory pain in the rat. Proc Natl Acad Sci USA 2007; 104(20): 8520-5.

[18] Dong XW, Goregoaker S, Engler H, et al. Small interfering RNAmediated selective knockdown of Nav 1.8 tetrodotoxin-resistant sodium channel reverses mechanical allodynia in neuropathic rats. Neuroscience 2007; 146(2): 812-21.

[19] Ekberg J, Jayamanne A, Vaughan CW, et al. muO-conotoxin MrVIB selectively blocks Nav 1.8 sensory neuron specific sodium channels and chronic pain behavior without motor deficits. Proc Natl Acad Sci USA 2006; 103(45): 17030-5.

[20] Bulaj G, Zhang MM, Green BR, et al. Synthetic muO-conotoxin MrVIB blocks TTX-resistant sodium channel Nav 1.8 and has a long-lasting analgesic activity. Biochemistry 2006; 45(23): 740414.

[21] Cummins TR, Waxman SG. Downregulation of tetrodotoxinresistant sodium currents and upregulation of a rapidly repriming tetrodotoxin-sensitive sodium current in small spinal sensory neurons after nerve injury. J Neurosci 1997; 17(10): 3503-14.

[22] Tanaka M, Cummins TR, Ishikawa K, Dib-Hajj SD, Black JA, Waxman SG. SNS Na+ channel expression increases in dorsal root ganglion neurons in the carrageenan inflammatory pain model. Neuroreport 1998; 9(6): 967-72.

[23] Priest BT, Murphy BA, Lindia JA, et al. Contribution of the tetrodotoxin-resistant voltage-gated sodium channel Nav 1.9 to sensory transmission and nociceptive behavior. Proc Natl Acad Sci USA 2005; 102(26): 9382-7.

[24] Rush AM, Waxman SG. PGE2 increases the tetrodotoxin-resistant Nav 1.9 sodium current in mouse DRG neurons via G-proteins. Brain Res 2004; 1023(2): 264-71.

[25] Burke JG, Watson RW, McCormack D, Dowling FE, Walsh MG, Fitzpatrick JM. Intervertebral discs which cause low back pain secrete high levels of proinflammatory mediators. J Bone Joint Surg Br 2002; 84(2): 196-201. 